\title{
Lower limb involvement in spinal function and low back pain
}

A. H. McGregor ${ }^{a *}$ and D W L Hukins ${ }^{\text {b }}$

${ }^{\text {a} B i o s u r g e r y ~ \& ~ S u r g i c a l ~ T e c h n o l o g y, ~ F a c u l t y ~ o f ~ M e d i c i n e, ~ I m p e r i a l ~ C o l l e g e ~}$ London, Charing Cross Hospital Campus, London, UK

${ }^{\mathrm{b}}$ School of Mechanical Engineering, University of Birmingham, Birmingham UK

*Address for correspondence:

Dr Alison McGregor

Biosurgery \& Surgical Technology,

Faculty of Medicine,

Imperial College London,

Charing Cross Hospital Campus,

London, W6 8RP

Tel: $+44(0) 2083838831$

Fax: $+44(0) 2083838835$

Email: a.mcgregor@imperial.ac.uk 


\section{Abstract.}

Aim: To evaluate evidence for involvement of the lower limb in spinal function and low back pain (LBP).

Design: A hypothesis based on a critical review of the relevant biomechanical and clinical literature.

Results: The spine resembles an inverted pendulum that supports the weight of the upper body; its stability requires a moving base that is provided by the joints of the lower limb, especially the hip. However, the sacroiliac joints are unlikely to be important for spinal function. The changing pattern of gait and development of lumbar lordosis, in early childhood, provide evidence for the inter-dependence of spinal curvature and lower limb action. Clinical signs associated with LBP may be associated with an inability to rotate the trunk about the hips. These include disorientation of the pelvis and weakness or tightness of muscles around the hip. The "sway back" posture seen in LBP involves flexion of the hip, knee and ankle to compensate for abdominal and back muscle weakness.

Conclusions: In order to understand the varied clinical presentation of LBP patients, the function of the spine should be considered in the context of the whole body, especially the lower limb.

Keywords: Clinical signs, low back pain, lower limb, sacroiliac joint, spine biomechanics 


\section{Introduction}

Despite low back pain (LBP) being a leading cause of long-term pain and disability, our understanding of its causes are poor and, consequently, its management is controversial [7]. Biomedical models of LBP have failed to link clinical assessment, diagnosis, treatment aimed and outcome [28]. "Biopsychosocial" models have gained widespread acceptance but have not led to higher success rates in managing LBP because they reflect a patient's psychosocial needs rather than providing insight into the causes of the problem [28].

Many biomedical models have been limited because they focus on specific structures rather than considering the spine as a dynamic system that is part of the whole body. Since the early work of Mixter and Barr [15], considerable emphasis has been placed on the intervertebral disc. This emphasis has been supported by the hypothesis that abnormal mechanics of the spinal column causes back pain [17]. Although abnormalities have been identified in the spine, e.g. degenerative or prolapsed discs, the relationship between these features, the resultant movement, pain and function is often unclear [3]. This suggests that our understanding of spinal function is deficient. The purpose of this paper is to present a range of evidence that suggests that the spine should not be considered in isolation from the lower limbs when trying to understand LBP. 


\section{Spinal mechanics}

Recently the spine has been modelled as an inverted pendulum in which a slender column supports the load of the upper body $[14,19,30]$. To maintain stability, an inverted pendulum requires a shifting base $[19,30]$. This condition for stability can be illustrated by balancing a pencil, point down, on a finger tip; when the pencil starts to fall, movement of the finger can reposition its centreof-gravity over the point restoring stability. In the spine this base is the pelvis and lower limbs; thus the spine can be stabilised, in the sagittal plane, by flexion of the hip, knee and ankle joints. For three-dimensional stability, lateral bending at some or all of these joints will be involved. This model of spinal function can rationalise many of the clinical features that LBP patients present with, and this will be explored in this paper.

In the past, the pelvis was believed to be a source of LBP and sciatica [11] with the sacroiliac joint $(\mathrm{SIJ})$ considered to be a source of pain and to have an important role in spinal function $[23,27]$. We believe that the SIJ is unlikely to be important because accurate measurement of its mobility by radiostereometric analysis (RSA) [24] shows that the standard deviations associated with movements at this joint are higher than the measurements themselves, i.e. there is no measurable movement. Further, our subjective experience is that manipulation, purported to mobilise the SIJ [22], really relaxes or alters the compliance of the soft tissues including the ligaments around the hip and SIJ. This experience is supported by interventional MRI 
studies of spinal mobilisations that do not realign the vertebrae but instead affect the surrounding soft tissues [13].

However, the spine is not a rigid vertical column (unlike the pencil in the example above) but is curved and capable of changing its curvature as a result of muscle action [1]. The concept of a Euler pendulum has been used to model the changes in curvature that occur e.g. in lifting [14]. During flexion, to support body weight and any additional weight carried, the spine is supported by muscles whose action is antagonistic to the muscles that initiated initial flexion, i.e. the spine is stiffened by active muscular contraction. Buckling theory shows that this stiffening can lead to changes in spinal curvature [14].

We suggest that movement at the hips and, perhaps to a lesser extent, at the knees and ankles can provide additional stability by moving the centre-ofgravity of the body with its supported weight, over the hips, i.e. along the centre-line in the sagittal plane. This proposed inter-dependence of spinal curvature and lower limb action is supported by evidence from children in the first 3 years after birth. Lumbar lordosis develops during this period, before the child starts to sit, stand or walk [20]. The child's initial gait is very different from that of an adult but changes as spinal curvature develops. Rotation about the hip provides a mechanism for translation of the trunk, during sitting, from a forward to a backward position that has been called the "lumbopelvic click clack movement" and attributed to movement at the SIJ [23]. 


\section{Clinical observations}

Clinical experience shows that patients with LBP often present with the following symptoms:

A. disorientation or mal-alignment of the pelvis, reflected in asymmetry in lower limb posture; - notably leg length inequality and compensatory changes at the feet i.e. pronation/supination and a resting asymmetry of the feet relative to the centre of gravity $[2,4,29]$

B. weak gluteal muscles [6]

C. tight hamstring and psoas muscles [5]

D. tight quadratus lumborum [5]

E. weak lower abdominal muscles including transversus abdominus [8]

F. restricted motion at the hip joint [26].

These observations are consistent with the involvement of the lower limb in spinal function, as described below.

Manipulative treatment to correct symptom $A$ is often supposed to mobilise the SIJ but is more likely to change the compliance of the soft tissues around the hip (see above), namely the anterior and posterior sacroiliac and iliolumbar ligaments and the iliopsoas, quadratus lumborum and piriformis muscles; all of these muscles contribute to rotation of the body around the hip joint [16]. Piriformis is associated with the sciatic nerve and along with the SIJ was one of the earliest recognised sources of sciatica [11] before attention shifted to the intervertebral disc. Postural leg length asymmetry and uneven positioning of the feet is likely to be adopted as a comfortable, and perhaps 
"lazy", way of supporting the upper body, especially when the spine and lower limbs are not functioning together in a coordinated manner. This lack of coordination could be caused by imbalances in strength and tension around the hip and pelvis. It may also result from the body's desire to maintain the eyes and head in a horizontal position regardless of the resulting postural imbalances it induces.

The gluteal muscles have an important role in maintaining pelvic alignment in the horizontal plane and are able to control rotation of the body about the hips. If there is weakness, the biceps femoris, a key hamstring muscle, compensates (symptom B). The biceps femoris and iliopsoas muscles both rotate the trunk about the hips [16]; tightness in these muscles (symptom C), a common finding in LBP, could be due to overuse or postural shortening and so provide evidence for the involvement of the hip in LBP (symptom F). Quadratus lumborum also tends to rotate the trunk about the hip, so tightness in this muscle (symptom D) has the same effect as tightness in hamstring and psoas muscles. In addition, when acting unilaterally, it changes the orientation of the pelvis, so its tightness is associated with symptom $A$ and may be a result of overuse to compensate for spinal problems.

Weakness in the abdominal muscles (symptom E) may be associated with the relationship between spine curvature and lower limb flexion, as well as having a direct influence on the efficiency of the spinal muscles. In "lower crossed syndrome" [5] hip flexors and the erector spinae tighten and shorten while the abdominal and gluteal muscles weaken; this leads to a "sway back" posture in 
which the hips, knees and ankles are flexed while the lumbar lordosis increases. Attempts to correct this posture focus on strengthening the lower abdominal and gluteal muscles and stretching the hamstrings and gluteal muscle groups. Contraction of the abdominal muscles will increase tension in the lumbodorsal fascia (LDF), whilst contraction of the gluteals influences tension in the ilio-tibial band. The LDF links the spine and pelvis and has been incriminated in LBP though its role remains poorly understood, mechanoreceptors have been indentified in its structure suggesting a role in motion and control [21]. In addition it is believed that the LDF assists the spine in lifting; there is no consensus on the mechanism involved $[9,10,12,18,25]$.

\section{Conclusions}

In conclusion, there is a range of evidence that the joints of the lower limb, especially the hip, are involved in spinal function and that they may be involved in LBP. The evidence that we have presented suggests that this involvement usually involves compensating for spinal dysfunction. However, it is equally possible that immobility of the hip and lower limb could lead to excessive spinal motion that could lead to LBP. There are two approaches to testing this hypothesis:

i) measuring inter-dependence of the spine and lower limbs in common tasks, or when the spine needs to return to a position of stability after sudden perturbation

ii) a prospective trial of the involvement of lower limb abnormality in compensation for or as a cause of LBP. 
The importance of this hypothesis is that it suggests that the spine should not be viewed in isolation from the lower limbs when considering the causes or treatment of LBP. 


\section{References}

1. R.M. Aspden, The spine as an arch. A new mathematical model. Spine 14 (1989), 266-274.

2. C. Barrey, J. Jund, O. Noseda and P. Roussouly, Sagittal balance of the pelvis-spine complex and lumbar degenerative diseases. A comparative study about 85 cases. Eur Spine J 16 (2007), 1459-1467.

3. M.C. Battié, T. Videman and E. Parent, Lumbar disc degeneration epidemiology and genetic influences. Spine 29 (2004), 2679-2690.

4. A.R. Bird, A.P. Bendrups and C.B. Payne, The effect of foot wedging on electromyographic activity in the erector spinae and gluteus medius muscles during walking. Gait Posture 18 (2003) 81-91.

5. L. Chaitow. Muscle Energy Techniques $2^{\text {nd }}$ edition, Churchill Livingstone, Edinburgh, 2001.

6. M.J. Comerford and S.L. Mottram. Movement and stability dysfunction-contemporary developments. Man Ther 6 (2001), 15-26.

7. European Commission Research Directorate General, Low Back Pain: Guidelines for its Management, Cost Action B13, http://www.backpaineurope.org/.

8. P.W. Hodges and C.A. Richardson. Inefficient muscular stabilization of the lumbar spine associated with low back pain. A motor control evaluation of transversus abdominis. Spine 21 (1996) 2640-2650.

9. D.W.L. Hukins, R.M. Aspden and D.S. Hickey. Thoracolumbar fascia can increase the efficiency of the erector spinae muscles. Clin Biomech 5 (1990), 30-34. 
10. D.W.L Hukins and R.M. Aspden, Thoracolumbar fascia. Clin Biomech 5 (1990), 241-242.

11. D. Lee, Clinical manifestations of pelvic girdle dysfunction, in: Grieve's Modern Manual Therapy $2^{\text {nd }}$ edition, J.D. Boyling and N. Palastanga, eds, Churchill Livingstone, Singapore, 1994, pp. 131-138.

12. J.E. Macintosh, N. Bogduk and S. Gracovetsky, The biomechanics of the thoracolumbar fascia. Clin Biomech 2 (1987), 78-83.

13. A.H. McGregor, P. Wragg and W.M. Gedroyc. Can interventional MRI provide an insight into the mechanics of a posterior-anterior mobilisation? Clin Biomech 16 (2001) 926-929.

14. J.R. Meakin, D.W.L. Hukins and R.M. Aspden, Euler buckling as a model for the curvature and flexion of the lumbar spine. Proc $R$ Soc Lond B 263 (1996), 1383-1387.

15. W.J. Mixter and J.A. Barr, Rupture of the intervertebral disc with involvement of the spinal canal. New England Journal of Medicine 211 (1934), 210-215.

16. K.L. Moore and A.F. Dalley, Clinically orientated anatomy $5^{\text {th }}$ edition, Lippincott Williams \& Wilkins, New York 2006.

17. M.M. Panjabi, T.R. Oxland, R.M. Lin and T.W. McGowen, Thoracolumbar burst fracture. A biomechanical investigation of its multidirectional flexibility. Spine 19 (1994), 578-585.

18. M.J. Pearcy, D.R.G. Williams and K.E. Moxham, Thoracolumber fascia. Clin Mech 5 (1990), 241.

19. N.P. Reeves, K.S. Narendra and J. Cholewicki, Spine stability: the six blind men and the elephant. Clin Biomech 22 (2007), 266-274. 
20. S. Reichmann and T. Lewin, The development of the lumbar lordosis. A post mortem study on excised lumbar spines. Arch Orthop Unfallchir 69: (1971), 275-285.

21. R. Schleip, A. Vleeming, F. Lehmann-Horn and W. Klingler. Letter to the Editor concerning "A hypothesis of chronic back pain: ligament subfailure injuries lead to muscle control dysfunction" (M. Panjabi). Eur Spine J 16 (2007), 1733-1735

22. K.A. Shearar, C.J. Colloca and H.L. White, A randomized clinical trial of manual versus mechanical force manipulation in the treatment of sacroiliac joint syndrome. J Manipulative Physiol Ther 28: (2005), 493501.

23. C.J. Snijders, P.F. Hermans, R. Niesing, C.W. Spoor and R. Stoeckart, The influence of slouching and lumbar support on iliolumbar ligaments, intervertebral discs and sacroiliac joints. Clin Biomech 19 (2004), 323329.

24. B. Sturesson, A. Uden and A. Vleeming, A radiostereometric analysis of the movements of the sacroiliac joints in the reciprocal straddle position. Spine 25 (2000) 214-217.

25. K.M. Tesh, J Shaw Dunn and J.H. Evans, The abdominal muscles and vertebral stability. Spine 9 (1984), 501-508.

26. L.R. Van Dillen, S.P. Gombatto, D.R. Collins, J.R. Engsberg and S.A. Sahrmann. Symmetry of timing of hip and lumbopelvic rotation motion in 2 different subgroups of people with low back pain. Arch Phys Med Rehabil 88 (2007), 351-360. 
27. M. Wang and G.A. Dumas, Mechanical behaviour of the female sacroiliac joint and influence of the anterior and posterior sacroiliac ligaments under sagittal loads. Clin Biomech 13 (1998) 293-299.

28. B. Weiner, Spine update: the biopsychosocial model and spine care. Spine, 33 (2008), 219-223.

29. D.K. Weiner, S. Sakamoto, S. Perera and P. Breuer, Chronic low back pain in older adults: prevalence, reliability, and validity of physical examination findings. J Am Geriatr Soc. 54 (2006), 11-20.

30. S. Zeinali-Davarani, H. Hemami, K. Barin, A. Shirazi-Adl and M. Parnianpour, Dynamic stability of spine using stability-based optimization and muscle spindle reflex. IEEE Trans Neural Syst Rehabil Eng 16 (2008), 106-118. 